\title{
Evolution of Stellar Population: Environments vs galaxy interactions
}

\author{
Josefa Perez ${ }^{1,2,3}$, Patricia Tissera ${ }^{1,2}$, Nelson Padilla ${ }^{4}$, Sol Alonso ${ }^{2,5}$ and \\ Diego G. Lambas ${ }^{2,6}$ \\ ${ }^{1}$ Instituto de Astronomía y Física del Espacio,Conicet-UBA, CC67, Suc.28, \\ Buenos Aires, Argentina. email: jperez fcaglp.unlp.edu.ar \\ ${ }^{2}$ Facultad de Ciencias Astronomía y Geofísica, Universidad Nacional de La Plata, Argentina \\ ${ }^{3}$ Consejo Nacional de Investigaciones Científicas y Técnicas, CONICET, Argentina. \\ ${ }^{4}$ Departamento de Astronomía y Astrofísica, Pontificia Universidad Católica de Chile, \\ Santiago, Chile. ${ }^{5}$ Complejo Astronómico El Leoncito, CP J5402DSP, San Juan, Argentina. \\ ${ }^{6}$ Observatorio Astronómico de la Universidad Nacional de Córdoba, Argentina.
}

\begin{abstract}
Several authors have studied the dependence of galaxy properties on environment in order to understand which mechanisms operate in the galaxy evolution. Recently, some of them have proposed that intermediate densities could be sites where local environment influences the transition of galaxies onto the red-sequence, as opposed to mechanisms that operate on cluster scales. Based on the evidence that interacting and merging systems are frequent at intermediate densities, we use the SDSS-DR4 data to analyse the role of close galaxy interactions as an environmental process which could contribute to lead evolutionary transformations. We explore the properties of galaxy pairs at different local and global density environments, comparing them with those of isolate galaxies in an unbiased control sample (CS).
\end{abstract}

Keywords. galaxies: evolution, galaxies: interactions, surveys.

\section{Galaxy Pair and Control Sample Catalogues}

From a reduced redshift range sample $(0.01<z<0.1)$ of the SDSS-DR4 galaxy survey and following observational criteria (Lambas et al 2003), we build a Close Galaxy Pair Catalogue (CGP) imposing thresholds in relative projected separations and radial velocities $\left(r_{p}<25 \mathrm{kpch}^{-1} ; \Delta V<350 \mathrm{~km} \mathrm{~s}^{-1}\right)$. For comparison, we build a CS constructed by selecting galaxies without a near companion within these thresholds. In order to avoid bias effects, it is required that galaxies in the CS match one-to-one redshifts, stellar masses, local densities and host halo masses of galaxies in pairs (Perez et al. 2009a). We also consider a merging systems (MS) sample, selecting galaxies from the CGP with clear morphological disturbances (Alonso et al. 2007).

The environmental analysis was done characterizing the local and global density regions respectively by, the projected density parameter, $\Sigma$, computed by using the projected distance to the $5^{\text {th }}$ nearest brighter neighbour, and the host halo mass, $M_{D M}$, assigned following Zapata et al. (2009). For this study, we divide the local and global environment in three different bins (see Table 1 of Perez et al. 2009b).

\section{Results and Conclution}

Computing the cummulative fraction of galaxies in CGP, MS and CS as a function of local projected density, we confirm previous results which show a larger frequence of interacting and merging systems at intermediate densities. We find that while $40 \%$ and $50 \%$ of close pairs and of merging systems, respectively populate the intermediate 
local densities, only a $25 \%$ of isolated galaxies in the CS are found in the same local environment (Perez et al. 2009b). This result inmediatly motivates the study of galaxy interactions at this particular moderate environment and their effects on the galaxy properties.

In order to separate the effect of galaxy interactions from other possible environmental processes, we compare the properties of galaxies with and without a near companion, inhabiting the same local and global environments. Particularly, we analyse colours $(u-r)$ and concentration indexes $\left(C=r_{90} / r_{50}\right)$ of galaxies in the CGP and CS, and inhabiting at the same local projected density $(\Sigma)$, in DM haloes of similar masses $\left(\mathrm{M}_{\mathrm{DM}}\right)$. In this comparison, we have removed any possible environmental bias, in consequence, we expect that properties of galaxies with and without a near companion behave similarly, unless tidal interaction effects operate.

The analysis of the fraccion of red $(u-r>2.8)$ and bulge-dominated systems $(C>2.5)$ as a function of local densities shows the expected underlying trends, with an increase of these fractions with $\Sigma$ for both isolated and interacting systems and regardless of the DM haloes. However, at intermediate local density regions, signals of galaxy interactions become recognized for all haloes. At these particular moderate local densities, trends of close galaxy pairs apart from those of isolated galaxies, with a larger fraction of red and bulge-like systems having a near companion (Perez et al. 2009b). Although, this excess of red-sequence objects in close pairs varies with $\mathrm{M}_{\mathrm{DM}}$, it is noticeable for all haloes suggesting that galaxies could be efficiently transformed at intermediate local densities by close galaxy interactions, process which might not be totally regulated by mechanisms which operate at large scales. We note that this red excess in close galaxy pairs might indicate that dust, stirred up during encounters, could partially obscure the tidally induced star formation (Gallazzi et al. 2008). However, other interpretations are also possible; i.e., galaxy interactions could operate on evolved systems. Infrared observations of galaxy pairs at moderate density regions could provide the key to better understand the problem.

\section{References}

Alonso, M. S., Lambas, D. G., Tissera, P., \& Coldwell, G. 2007, MNRAS 375, 1017

Gallazzi, A., Charlot, S., Brinchmann, J., White, S. D. M., \& Tremonti, C. A. 2005, MNRAS 362,41

Lambas, D. G., Tissera, P. B., Alonso, M. S., \& Coldwell, G. 2003, MNRAS 346, 1189

Perez, J., Tissera, P., \& Blaizot, J. 2009, MNRAS 397, 748 (Paper a)

Perez, J.,Tissera, P., Padilla, N., Alonso, S., \& Lambas, D. G. 2009, in publication by MNRAS (Paper b, astroph/0904.2851)

Zapata, T., Perez, J., Padilla, N., \& Tissera, P. 2009, MNRAS , 394, 2229 